\title{
Emotional Evoked Analysis of Classical Music
}

\author{
Ying Lin \\ Weinan Normal University, Weinan, Shaanxi, 714000
}

\author{
Keywords: Classic Music, Emotional Evoked Analysis, Control Charts
}

\begin{abstract}
EEG induced emotional music and autonomic nervous response. Methods A total of 20 graduate students ( 8 boys and 12 girls) were randomly selected and listened to two melodies with the same melody but played with different instruments. The BIOPAC MP150 multi-guided physiology instrument was used to analyze the subjective experience and electroencephalogram Autonomic and physiological responses were studied. Results (1) The happy and sad emotions were induced by the two pieces of music $(\mathrm{t}=8.90, \mathrm{P}<0.01 ; \mathrm{t}=6.47, \mathrm{P}<0.01) 0.037)$; (3) The maximum energy of $\mathrm{T}$ wave, $\mathrm{U}$ wave and $\theta$ wave were enhanced in different brain regions under the two different emotions. Conclusions The maximal energy of $\mathrm{T}$ wave, $\mathrm{U}$ wave and $\theta$ wave are significantly affected by emotion. Pleasant emotions activate $\mathrm{T}$ wave in the occipital region and the apical region more, and sad mood more activates the $\mathrm{T}$ wave in the frontal region.
\end{abstract}

\section{Introduction}

In recent years, psychologists have paid special attention to the research of autonomous physiological responses to emotions because of the obvious connection between these independent physiological changes and emotions. One of the underlying hypotheses in emotional EEG studies is that increased alpha activity at $8-13 \mathrm{~Hz}$ is associated with weakened cortical activity in this cortical area, so that as alpha rhythms become desynchronized, it can be inferred that presence in this area A strong cognitive or emotional processing [1]. Some researchers have found that $\beta$ activity, especially $\beta$ activity in the temporal lobe, is also involved in emotional processing. Not only that, the $\theta$ rhythm involves the processing of emotions and cognitions. Stenberg found that the increase in theta activity was associated with "visceral pleasures," and that in the right lateral forehead, a nearly-verge position recorded on the scalp was found near the limbic system and showed a strong increase in theta activity when induced, Intracranial stimulation of the cingulate leads to "euphoria" and also enhances $\theta$ rhythmic activity [2]. Evoked stimulus studies of previous emotions found that the means of inducing emotions varied, including visual, auditory, memory and so on. Phan et al made a review and found that the use of auditory stimulation induced sentiment rarely [3]. However, music is an important auditory stimulus. Some foreign studies on music and emotion use the musical stimuli as the symphony or the music in the opera. Domestic physiological researches on emotion are scarcer. In traditional Chinese art, music can best embody the meaning of life in Chinese art. Chinese classical music particularly emphasizes nature. Therefore, classical Chinese music has its own unique advantages in mobilizing the emotions. It is possible that the study of the relationship between classical Chinese music and emotion may have implications for China The traditional regimen and psychotherapy provide the theoretical basis.

\section{Objects and Parties}

A total of 20 graduate students (8 boys and 12 girls) were selected at random. The average age was 22-28 years old (25 \pm 1.30 years). All subjects were right-handed with no brain disease. The experiment is 2 (Yun, zheng) $\times 2$ (male, female) factor experimental design.

Experimental materials using Plutchik 5-point emotional rating scale, the general questionnaire and hand questionnaire. Music using classical Chinese music "Yang Guan Triad." In addition to singing as a song, Yang Guan Triad often performs instrumental music. Among them, Qin Song, Zheng Music, Erhu Music and Qu Opera have more influence. The experiment is the use of Zheng 
music and song, each song is solo.

Experimental Procedures and Tasks The design of this experiment required that each participant listen to music and zheng music. Before the experiment, we arranged the listening order of the subjects and adopted the AB-BA offsetting method to avoid the order effect. The subjects only listened to a piece of music once a week and listened to the second piece at the same time after one week. After being tested in the lab, fill out the questionnaire and emotion scale. Multi-channel physiology was turned on, stimulating the subjects' physiological indexes while playing white noise for 1 minute and then playing the music (Zheng music). The music is rendered as a 1-minute white noise as a recovery value. After the second phase of white noise was completed, the experimental program was closed and subjects were asked to fill in the emotional scale and the general questionnaire. In response to questions raised by the subjects, subjects were asked to conduct a second experiment at the same time one week later. The second experiment is the same as the first experiment, except that the second experiment is listening to Zheng music (Yun music). In this experiment, BIOPAC MP150 system was used to measure two physiological indexes of EEG and skin electricity. All the data were presented on the main computer. Take the first 5 minutes of each piece of music data, take a 1 minute after the white noise data as a comparison, using SPSS 10.0 analysis of variance.

Discrepancy test of subjective experience The results of paired t-test showed that subjects' subjective experience was influenced only by the type of music and not by gender. Multivariate analysis of variance also showed that when listening to music, happy scores decreased significantly, sadness scores increased significantly, while other emotions did not differ significantly; while listening to music, happy scores increased significantly, while other emotions did not differ significantly.

Multivariate analysis of variance showed that the main effect of music type was not significant $(\mathrm{F}=0.882, \mathrm{P}=0.360)$, and the main effect of gender was significant $(\mathrm{F}=16.82, \mathrm{P}=0.001), \mathrm{P}=$ 0.668). This shows that the change of skin electricity is affected by gender without being affected by the type of music. Music type in the occipital area, forehead F7F8 channel, the main effect of the temporal area, the type of music for further testing, the results found: Yun music state and Zheng music state compared to the occipital area, frontal F7F8 channel, temporal $\alpha$ wave significant difference. This shows that in the three channels of the three brain regions, the alpha wave energy in the music state is higher than the alpha wave energy in the zheng music state.

\section{Results discussion}

According to the statistics of emotion questionnaire, when the participants listened to the music piece, the scores of sadness increased significantly, and the happy score decreased significantly, which indicated that the music piece successfully induced the sad mood of the participants and reduced the happy mood of the subjects; When the happy score significantly increased, indicating that Zheng music successfully induced the participants happy mood, fully proved that different timbre music can induce different kinds of emotions. Further tests found that subjects' subjective experience was influenced only by the type of music and not by gender. The subjective experience shows that there is no gender differences, which is consistent with the results of previous studies.

The two kinds of emotions have little effect on the skin electricity, but different gender test subjects skin electrical response is different. In both sad mood and happy mood, the skin electricity of male subjects was higher than that of female subjects. This shows that the two emotions induced by this experiment have no effect on the skin electricity. The experiment found that: in frontal area, temporal area and occipital area, sadness, alpha wave energy than the happy mood of alpha wave energy. Sadness activated the frontal area, the temporal area and the occipital area, which is inconsistent with the results of Jia Jing's study [5]. We speculate that the reason for the inconsistency may be related to the stimulus material. In the occipital, frontal and temporal regions, the $\beta$-wave energy under sad emotion is higher than the $\beta$-wave energy under the pleasant emotion. This shows that sadness, in the occipital, frontal and temporal regions of the maximum energy $\beta$ wave significantly increased, the results of Helen's findings similar. The test of $\theta$ wave showed that 
in the central area and the apex area, emotion had a significant influence on the $\theta$ wave, and reached a significant level in the occipital and temporal areas, which was consistent with previous studies. In the occipital, apical and temporal regions, emotions and sex have an interaction. There was a difference in $\theta$ wave energy between men and women, so we could not mix the results of men and women with statistics. This is the same as previous studies. In pure emotional state, sadness caused the $\theta$ wave in the temporal area to decrease significantly, while the pleasant emotion caused the $\theta$ wave in the occipital area to decrease significantly.

Relative to the emotional and research, multi-guided physiology has its obvious advantages, it is mainly research, but also the study of skin electricity, heart rate, etc., these indicators are emotional physiological response is essential. Which is the use of high-sensitivity biological signal amplifier, the nerve cell spontaneous electrical activity received, amplified, traced curve, it is a smooth, regular and recurring curve. Because nerve cells have different activity patterns, the traced brain waves have four rhythms under normal conditions. They are uniformly named as wave one, wave one, wave one, wave one and wave. . And is fast wave, most healthy people record to EEG most of them, called. And dominant. The study on the basis of emotion and physiology is more abroad, while the study in China is relatively weak. In this study, we selected college students as subjects and asked the subjects to listen to the same piece of music played by different instruments in two different times. Before and after the experiment, we filled in the emotional questionnaire and tried to induce two different emotions with two different timbre songs and sought emotional and Skin electricity, skin temperature and heart rate and other autonomic neurophysiological indicators of the relationship between emotions and different brain regions of the wave.

According to the statistics of emotion questionnaire, when the participants listened to the melody, the scores of grief increased significantly, and the scores of happiness decreased significantly, which indicated that the melody successfully induced the sad mood of the participants and reduced the pleasure of the participants. Happy score increased significantly, indicating that Zheng music successfully induced the participants happy mood. The difference between the two emotions is significant, namely, the two kinds of music not only induce two completely different emotions, but also the two emotions are completely different in the subjective experience. This verifies the previous study of music and emotion, which fully proves that different timbre music can induce different kinds of emotions. Zheng music can induce happy emotions, and music can induce sad emotions. Further examination revealed that subjects' experience of subjective experience was influenced only by the type of music and not by gender. This shows that there is no gender difference in subjective experience. Subjective experience is the same when subjects of different sexes listen to the same type of music, which is consistent with the results of previous studies. The subjects after the experiment survey found that the participants agreed that the music gives the feeling of music is sad, desolate, Ruqirusu Zheng and Zheng music is to give people melodious, serene, cheerful. All these prove that the two different timbre selected in this experiment have different subjective experiences.

The statistics found that there was no significant difference in main effects of oxygen saturation, respiratory rate and respiration rate, and there was no interaction between genres and genres of music. This shows that neither the genre of music nor gender has any influence on these three physiological indices, and their changes have not been influenced by experimental design. In the past, we did not adopt the sentiment study of these three indicators. Therefore, we can not compare them. Although they did not change significantly in this experiment, we can not say that there is no relationship between the emotion and the oxygen saturation, respiration rate and respiration rate. Skin electricity is the first indicator of basic research in early emotional physiology. And so found fear, disgust more than happy lead to greater skin conductivity. The study found that the two types of emotions have little effect on the skin electricity, but different gender test skin electrical response is different. Under the sad mood, the skin electricity of male subjects was higher than that of female subjects, and the skin electricity of male subjects was also higher than that of female subjects. However, the sad mood compared with the happy mood, whether male or female test subjects, skin electricity did not change significantly, which also shows that the two emotions induced by this 
experiment had no effect on the skin electricity. This is not exactly the same as the forecast.

\section{Conclusion}

This article for the first time combined with the autonomic nervous response to emotional brain physiology basic research, and has been more valuable results, but due to time, energy and technical limitations, or left some regret The study of each band is not meticulous In music stimulation Aspects, the selected music is not broad enough. These will be the direction for our future efforts. We hope that in future experiments, we will do further analysis of each wave and divide them into narrower bands for research. When selecting music, we can select some Chinese folk music and some foreign music to combine other neuroimaging techniques, The result is verified. Due to the limitations of instrumentation, fewer leads were used in this study, and we hope to use more leads to record EEG in future studies.

\section{Acknowledgements}

Weinan Teachers College of Education Science International Cooperation Project (17GJHZ22)

Shaanxi Province in 2017 military and civilian integration research fund project (17JMR06)

Weinan Teachers College Specialty Discipline Project (14TSXK12)

\section{References}

[1] Barlow J S. The Electroencephalogram: its Patterns and Origins [J]. MIT Press Cambridge MA, 1993, 12 (1): 2256-2268

[2] Stenberg G. Personality and the EEG: Arousal and emotional arous-ability [J]. Pers Individ Differ, 1992, 13 (5): 1097-1113

[3] K Luan Phan, Tor Wager and Israel Liberzon. Functional Neu-roanatomy of Emotion: A MetaAnalysis of Emotion Activation Studies in PET and fMRI [J]. Neuron Image, 2002, 16 (1): 331-348

[4] Hubert W, de Jong-Meyer. Psychophysiological response pattern sto positive and negative film stimuli [J]. Biological Psychology, 1990, 31 (1): 73-93

[5] Jia Jing, Liu Chang. .Research on the physiological activities induced by video clips [J] .Chinese Journal of Health Psychology, 2008, 16 (10), 1187-1189

[6] Helen JC. Self-generated happy and sad emotions in low and high-ly hypnotizable persons during waking and hypnosis: laterality and regional EEG activity differences [J]. International Journal of Psy-chophysiology, 1996, 24: 239-266

[7] Schellberg D, Besthorn C, Klos T, et al. EEG power and coherencewhile male adults watch emotional video films [J]. Psychophysiol, 1990, 9 (1): 279-291 